\title{
DIVERSIDADE DA ICTIOFAUNA DE POÇAS DE MARÉ DA PRAIA DO CABO BRANCO, JOÃO PESSOA, PARAÍBA, BRASIL
}

\author{
Ricardo S. Rosa ${ }^{1}$ \\ lerecê L. Rosa ${ }^{1}$ \\ Luiz A. Rocha ${ }^{1}$
}

\begin{abstract}
DiVERSITY OF THE TIDAL POOL ICHTHYofaUna OF CABO BRANCO BEACH, JoÃo Pessoa, Paraíba, Brazil. A survey of the tidal pool fish community was carried out between february/1987 and january/1988, at Cabo Branco Beach, João Pessoa, Paraíba, Brazil. Specimens were collected monthly at low tide, using rotenone. A total of 3143 specimens was obtained. The most speciose families were Ophichthidae ( $14 \%$ of the total number of species), Mugilidae (11\%) and Muraenidae ( $9 \%)$, and the most representative ones in number of individuals were Gobiidae (48\%), Pomacentridae $(13 \%)$ and Labrisomidae (10\%). The main resident species were Bathygobius soporator (Valenciennes, 1837) and Abudefduf saxatilis (Linnaeus, 1758). The presence of a large number of juveniles in the pools indicated the importance of the studied sites as nursery areas for several fish species.

KEY WORDS. Ichthyofauna, diversity, Paraiba, Brazil
\end{abstract}

A zona entre-marés, situada entre as linhas de preamar e baixamar, separa-se aproximadamente duas vezes ao dia do mar aberto, durante a baixamar. A água remanescente pode formar pocas de maré que, durante a preamar, religam-se ao mar e tornam-se sujeitas à acão das ondas e da turbulência (HORN \& GIBSON 1988). As poças de maré constituem-se em habitat específico para numerosas espécies de peixes, servindo ainda como áreas de criadouro de larvas e jovens de várias outras espécies (CRABTREE \& DEAN 1982).

Durante a baixamar, quando os peixes se concentram em seu interior, as poças de maré tornam-se ambientes adequados para a amostragem e estudo quantitativo da ictiofauna. A maioria dos trabalhos recentes têm usado o ictiotóxico rotenona para esta finalidade (LARDNER et al. 1993). RANDALL (1963) considera a rotenona como o mais valioso método para a coleta científica de peixes. Esta técnica também é propícia para estudos de recolonização dos nichos desocupados (LARDNER et al. 1993).

A composição da ictiofauna e os padrões de diversidade, distribuição e abundância de espécies de peixes em poças de maré foram estudados em diversas áreas, sobretudo de regiões temperadas, como a costa da Califórnia (FITCH \&

Departamento de Sistemática e Ecologia, Centro de Ciências Exatas e da Natureza, Universidade Federal da Paraíba. 58059-900 João Pessoa, Paraíba, Brasil.

E-mail: cendse09@brufpb.bitnet. 
LAVENBERG 1975; YoshiYAMA 1981; Moring 1986; YoshiYAMA et al. 1986), Taiwan (LEE 1980), África do Sul (BENNETT \& GRIFFITHS 1984; BECKLEY 1985; BENNETT 1986) e New South Wales (LARDNER et al. 1993). No Brasil, levantamentos ictiofaunísticos de poças de maré foram essencialmente qualitativos (STARKS 1913; ALMEIDA 1973).

A ponta do Cabo Branco, João Pessoa, Paraíba, caracteriza-se pela presença de grande quantidade de rochas areníticas, ferruginosas ou não, que se estendem do litoral até centenas de metros mar adentro. Pela ação das ondas, essas rochas são erodidas e fragmentadas, resultando em um acúmulo de pedras em vários pontos da praia, que, juntamente com algas calcárias, delimitam poças de maré.

Neste trabalho, selecionamos dois conjuntos de poças de maré da ponta do Cabo Branco e neles amostramos a ictiofauna, objetivando conhecer sua composição específica e a distribuição relativa e abundância das espécies ao longo do ano.

\section{MATERIAL E MÉTODOS}

\section{Área de estudo}

A área de estudo (Fig. 1) situa-se no extremo sul da Praia do Cabo Branco, João Pessoa, Paraíba (0709' $16^{\prime \prime}$ S; 34 48 '17" W), onde dois pontos de coleta foram estabelecidos. O ponto "A" (Fig. 2 ) consiste de uma poça de $37 \mathrm{~m}$ de comprimento, $18 \mathrm{~m}$ de largura e $40 \mathrm{~cm}$ de profundidade máxima, distando $80 \mathrm{~m}$ da linha de baixamar, medida na cota 0,1 . Seu substrato é constituído por areia e rochas, parcialmente recoberto por várias espécies de macroalgas (Solieria sp, Vidalia sp., Gracilaria spp., Hypnea musciformis(Wulfen) Lamouroux, Padina sp, Dictyopteris spp. e Ulva lactuca Linnaeus). O ponto "B" (Fig. 3) é formado por duas poças ovaladas, com comprimento de 6,0 e 4,0 m, largura máxima 3,40 m e profundidade máxima de $15 \mathrm{~cm}$, distando $60 \mathrm{~m}$ da linha de baixamar, medida na cota 0,1 ; seu substrato é predominantemente rochoso. Quanto à sua situação espacial, os dois conjuntos de poças podem ser caracterizados como litorais, segundo a classificação de Doty (1957).

Foram efetuadas duas coletas mensais em condições de baixamar, uma diurna e uma noturna, no período de fevereiro de 1987 a janeiro de 1988, com ictiotóxico à base de rotenona (Nusyn-Noxfish, Penick Corporation). A amplitude máxima das marés durante o período de estudo foi de $2,7 \mathrm{~m}$, segundo a tábua de marés da Diretoria de Hidrografia e Navegação para o porto de Cabedelo, PB. A temperatura da água foi registrada no início de cada coleta com termômetro de mercúrio. Os exemplares capturados foram fixados em solução de formol a $10 \%$ tamponada com borato de sódio e preservados em álcool etílico $75^{\circ} \mathrm{GL}$. Os espécimes foram identificados utilizando-se a literatura especializada e sua apresentação nos resultados segue a classificação de famílias de NELSON (1994). O material ictiológico encontra-se depositado na coleção ictiológica da Universidade Federal da Paraíba, João Pessoa (UFPB).

Para caracterizar a diversidade da comunidade de peixes dos locais estudados, utilizou-se o índice de Shannon-Wiener $\left(H^{\star}\right)$, um índice matemático que expressa a relação entre o número de espécies e sua abundância relativa (THORNE- 


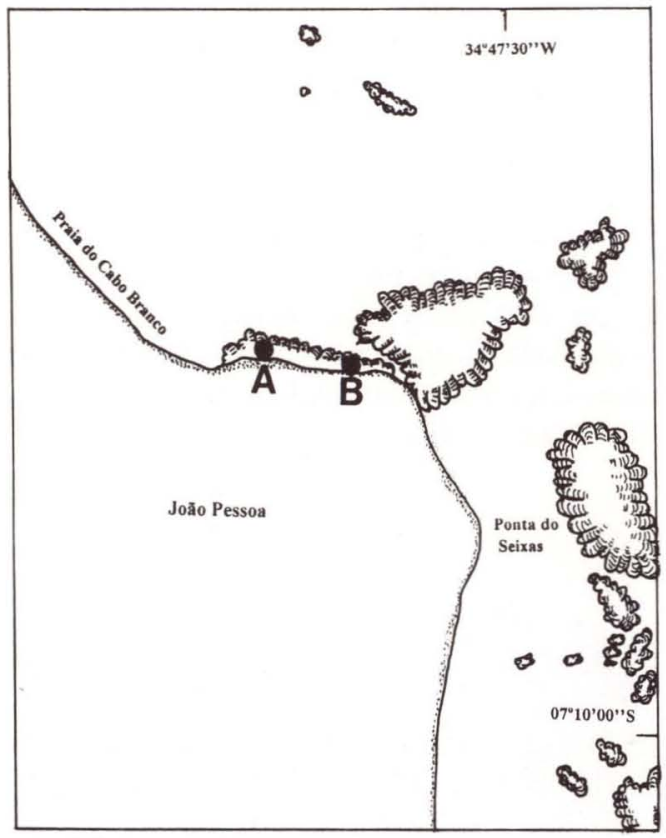

Fig. 1. Área de estudo, mostrando os pontos de coleta "A" e "B".

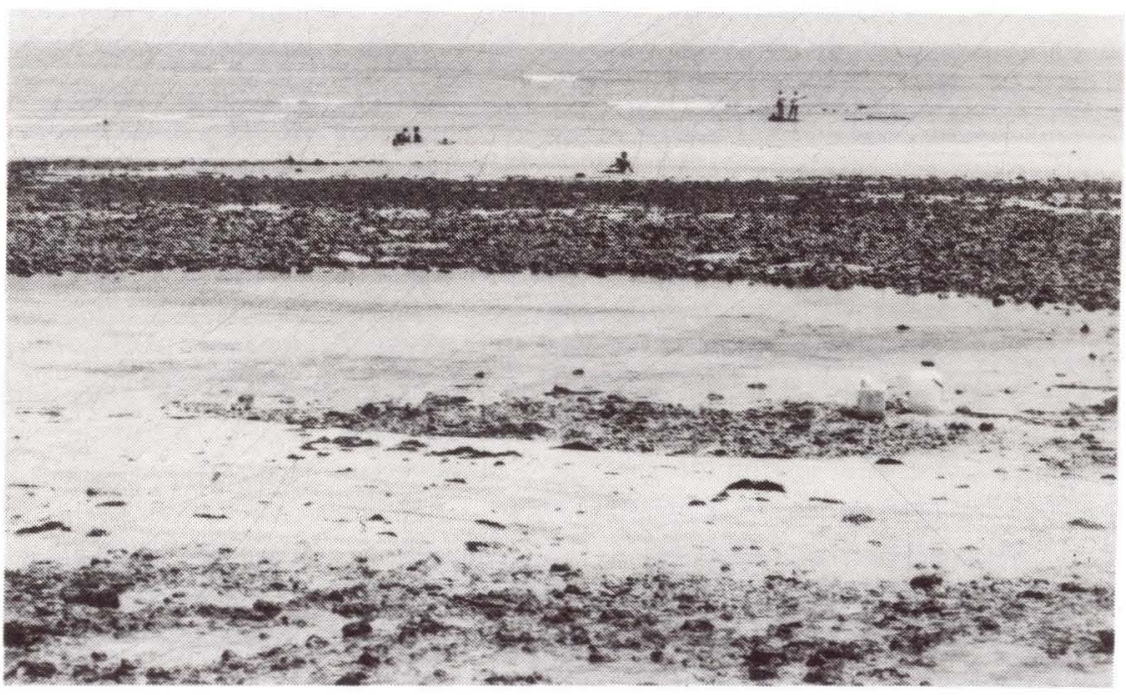

Fig. 2. Vista parcial do ponto de coleta "A", Praia do Cabo Branco, João Pessoa, Paraíba. 
MiLlER \& CATENA 1991). Sua expressão matemática é a seguinte:

$$
H^{\prime}=-\sum_{i=l}^{s}\left[\left(\frac{n i}{n}\right) \ln \left(\frac{n i}{n}\right)\right]
$$

onde $n_{i}$ é o número de indivíduos pertencentes à $i$-ésima espécie de $S$ espécies da amostra e $n$ é o número total de indivíduos da amostra. Segundo MARGALEF (1986), é uma função que apresenta baixa sensibilidade à extensão da amostra, com a possibilidade de ser utilizada em populações de tamanho desconhecido. Este índice, na forma expressa acima, é um dos mais amplamente utilizado por biólogos (BAKUS 1990; LUDWIG \& REYNOLDS 1988).

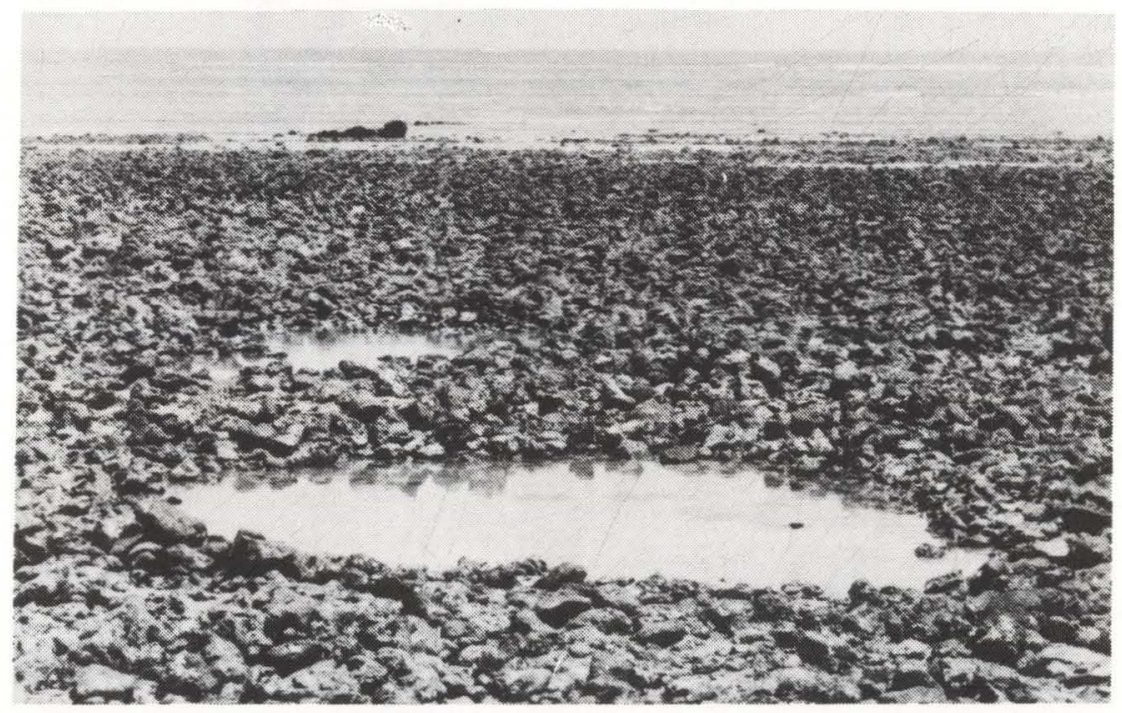

Fig. 3. Vista parcial do ponto de coleta "B", Praia do Cabo Branco, João Pessoa, Paraiba.

O índice de Shannon-Wiener é influenciado tanto pelo número de espécies quanto pelo número de indivíduos (de cada espécie) da amostra, sendo ele o produto da interação desses dois fatores. O número de espécies da amostra é denominado "riqueza de espécies" e a distribuição dos indivíduos dentro de cada espécie "equitabilidade" (KRICHER 1972). Para o cálculo deste índice foi utilizado o programa COMM, desenvolvido para microcomputadores padrão IBM e apresentado por PIEPENBURG \& PIATKOWSKI (1992).

Para caracterizar a ocupação das poças pelos peixes, foram estabelecidas as seguintes categorias (modificadas de CRABTREE \& DEAN 1982): residentes primários - espécies presentes durante todo o ano como jovens e adultos; residentes secundários - espécies presentes o ano todo, mas apenas como jovens; visitantes ocasionais - espécies que ocorrem em ambientes adjacentes e entram nas poças durante a maré alta; raras - espécies que ocorrem esporadicamente nas poças. 


\section{RESULTADOS E DISCUSSÃO}

As espécies obtidas e o número de exemplares coletados por mês encontramse listados nas Tabelas I-IV. Um total de 3143 indivíduos, pertencentes a 44 espécies e a 22 famílias, foi obtido nos dois pontos de coleta. O ponto "A" contribuiu com 2355 indivíduos e 41 espécies, e o ponto "B" com 788 indivíduos e 18 espécies; 15 espécies estiveram presentes nos dois pontos. As coletas diurnas contribuíram com 1179 indivíduos capturados e as coletas noturnas com 1964.

Tab. I. Lista das espécies de peixes da região entre-marés da Praia do Cabo Branco, João Pessoa, Paraíba, com o número de exemplares coletados entre fevereiro de 1987 e janeiro de 1988 (Ponto "A", coletas diurnas).

\begin{tabular}{|c|c|c|c|c|c|c|c|c|c|c|c|c|}
\hline Espécies & Fev & Mar & $\mathrm{Abr}$ & Mai & Jun & Jul & Ago & Set & Out & Nov & Dez & Jan \\
\hline Albula vulpes (Linnaeus, 1758) & & & & & & & & & 1 & & & 1 \\
\hline Myrichthys ocellatus (Le Sueur, 1825) & & & & & 1 & & & 1 & & & 1 & \\
\hline Ahlia egmontis (Jordan, 1884) & 2 & & & 1 & & & & & & & & \\
\hline Myrophis punctatus Lütken, 1851 & & & & & 1 & & 1 & & 1 & & & 3 \\
\hline Bascanichthys paulensis Storey, 1939 & & & & & & & & & & & & 2 \\
\hline Callechelys sp. & 1 & & & & & & & & & & & \\
\hline Gymnothorax funebris Ranzani, 1840 & 1 & & & & & & & 1 & & 1 & & \\
\hline Gymnothorax vicinus (Castelnau, 1855) & 1 & & & & & & & & & & & \\
\hline Gobiesox strumosus Cope, 1870 & 1 & & & & & & & 1 & & & & \\
\hline Lutjanus jocu (Bloch \& Schneider, 1801) & 3 & 2 & & 1 & & & 4 & & 2 & 2 & 2 & 3 \\
\hline Lutjanus apodus (Walbaum, 1792) & 2 & 5 & 1 & 1 & & & 1 & & 3 & & 28 & 25 \\
\hline Eucinostomus melanopterus (Bleeker, 1863) & & & & & & & & & & & 2 & 1 \\
\hline Eucinostomus sp. & & & & 4 & & & & & & & & \\
\hline Stegastes variabilis (Castelnau, 1855) & & & & & & & & & & 3 & & 1 \\
\hline Abudefduf saxatilis (Linnaeus, 1758) & & & 1 & & 1 & 3 & 7 & 37 & 2 & & & 2 \\
\hline Mugil liza Valenciennes, 1836 & & & & & & & 6 & & 3 & & & 3 \\
\hline Mugil gaimardianus Desmarest, 1831 & & & & & & & & 32 & 1 & & & \\
\hline Mugil sp. & & & & & & & 1 & & & & & \\
\hline Polydactylus virginicus (Linnaeus, 1758) & & & & & & & 1 & & & & & \\
\hline Sparisoma rubripinne Valenciennes, 1839 & & 1 & & & & & & 1 & & & & 13 \\
\hline Sparisoma radians (Valenciennes, 1839) & 1 & & & & & & & & & & & \\
\hline Labrisomus nuchipinnis (Quoy \& Gaimard, 1824) & 14 & 10 & & & 2 & & 2 & & 1 & & 1 & \\
\hline Malacoctenus delalandii (Valenciennes, 1836) & 5 & & & & & & & & & & & \\
\hline Scartella cristata (Linnaeus, 1758) & 2 & & & 4 & 8 & & 7 & & & & & \\
\hline Gobionellus boleosoma (Jordan \& Gilbert, 1882) & 1 & 6 & 3 & & & & & 6 & 3 & 4 & 4 & 2 \\
\hline Bathygobius soporator (Valenciennes, 1837) & 85 & 25 & 53 & 64 & 42 & 37 & 95 & 60 & 7 & & 7 & 6 \\
\hline Acanthurus chirurgus (Bloch, 1787) & & & & & & & 1 & & & & & \\
\hline Achirus achirus (Linnaeus, 1758) & & & & & & & & & & 1 & & \\
\hline Sphoeroides testudineus (Linnaeus, 1758) & & & & & & & & 2 & & & 3 & 4 \\
\hline
\end{tabular}

Apesar da diferença significativa no nível de $5 \%$ (qui-quadrado estimado $=$ 6,25 ) entre os totais de indivíduos capturados durante o dia e à noite, consideramos que estes resultados foram possivelmente afetados pela proximidade temporal entre coletas diurnas e noturnas, já que em oito dos 12 meses amostrados, as coletas noturnas precederam as diurnas por um intervalo máximo de quatro dias. Embora a recolonização após o uso de rotenona pareça ocorrer de modo rápido, o seu uso pode ter causado uma depleção faunística nas poças estudadas, sem que tenha havido a reocupação de todos os nichos vagos entre as coletas. Observou-se que, na única vez em que as coletas diurna e noturna foram realizadas no mesmo dia, houve uma considerável depleção no contingente da espécie residente mais abundante ( $\mathrm{Ba}$ - 
Tab. II. Lista das espécies de peixes da região entremarés da Praia do Cabo Branco, João Pessoa, Paraíba, com o número de exemplares coletados entre fevereiro de 1987 e janeiro de 1988 (Ponto "A", coletas noturnas).

\begin{tabular}{|c|c|c|c|c|c|c|c|c|c|c|c|c|}
\hline Espécies & Fev & Mar & Abr & Mai & Jun & Jul & Ago & Set & Out & Nov & Dez & Jan \\
\hline Albula vulpes (Linnaeus, 1758) & & & & & & & & & 1 & 1 & & \\
\hline Ahlia egmontis (Jordan, 1884) & & & & & & & 1 & 3 & & & & \\
\hline Myrophis punctatus Latken, 1851 & & & 1 & & & & 2 & 2 & 1 & 6 & 7 & 5 \\
\hline Myrichthys ocellatus (LeSueur, 1825) & 6 & & & 1 & & & 2 & 2 & & 3 & 1 & 2 \\
\hline Bascanichthys paulensis Storey, 1939 & 3 & & & & & & 3 & 15 & 1 & 6 & 7 & 5 \\
\hline Gymnothorax funebris Ranzani, 1840 & 2 & 1 & & 1 & & & & 5 & & 2 & & \\
\hline Gymnothorax vicinus (Castelnau, 1855) & & & & & & & 2 & & & & 1 & \\
\hline Gymnothorax moringa (Cuvier, 1829) & & & 1 & & & & 1 & & & & & \\
\hline Gobiesox strumosus Cope, 1870 & 1 & & & & & & & & & & & \\
\hline Holocentrus ascencionis (Osbeck, 1765) & & & & & & & & & & 2 & & \\
\hline Epinephelus adscencionis (Osbeck, 1771) & & & & & & & 1 & 1 & 1 & 1 & & \\
\hline Caranx latus Agassiz, 1831 & & & & & & & & & & 5 & & \\
\hline Trachinotus falcatus (Linnaeus, 1758) & & & & & & & & & & 1 & & \\
\hline Lutjanus jocu (Bloch \& Schneider, 1801) & 2 & 10 & 4 & 5 & 2 & 1 & 2 & 8 & & 19 & 1 & 4 \\
\hline Lutjanus apodus (Walbaum, 1792) & & 3 & & 8 & 1 & & 2 & 8 & 3 & 28 & 14 & 25 \\
\hline Eucinostomus argenteus Baird \& Girard, 1854 & & & & & & & 5 & 3 & & 2 & 3 & \\
\hline Eucinostomus melanopterus (Bleeker, 1863) & 1 & & & 2 & & & & 2 & & 9 & 54 & 16 \\
\hline Haemulon steindachneri (Jordan \& Gilbert, 1882) & & & & & & & 1 & & & & & \\
\hline Umbrina coroides (Cuvier, 1830) & & & & & & & & 7 & & & & \\
\hline Abudefduf saxatilis (Linnaeus, 1758) & 2 & & 11 & 99 & 37 & & 29 & 17 & 2 & 9 & & 2 \\
\hline Mugil liza Valenciennes, 1836 & 1 & & & 2 & & & & & & & & \\
\hline Mugil gaimardianus Desmarest, 1831 & & & & & & & 8 & 7 & 3 & & & \\
\hline Mugil trichodon Poey, 1876 & & & & & & & & & & & 1 & \\
\hline Mugil curema Valenciennes, 1836 & & & & & & & 3 & 11 & 1 & & & \\
\hline Mugil sp. & & & & & & & & & 1 & 1 & & \\
\hline Polydactylus virginicus (Linnaeus, 1758) & & & & & & & 3 & 18 & & 5 & & \\
\hline Sparisoma rubripinne Vallenciennes, 1839 & 1 & & 1 & & 1 & 73 & 3 & 5 & & & 2 & \\
\hline Stegastes variabilis (Castelnau, 1855) & & & & & 4 & 21 & 3 & & & & & \\
\hline Anisotremus surinamensis (Bloch, 1791) & 8 & & 1 & & & 2 & 1 & & & & & \\
\hline Labrisomus nuchipinnis (Quoy \& Gaimard, 1824) & 30 & 6 & 12 & 5 & 22 & 60 & 23 & 19 & 9 & 15 & 3 & 1 \\
\hline Malacoctenus delalandii (Valenciennes, 1836) & 8 & & & & & & & & & & & \\
\hline Scartella cristata (Linnaeus, 1758) & & & & 1 & 2 & & & & & 2 & 3 & \\
\hline Gobionellus boleosoma (Jordan \& Gilbert, 1882) & 1 & 6 & 1 & & & & & & 2 & 17 & 10 & 10 \\
\hline Bathygobius soporator (Valenciennes, 1837) & 40 & 9 & 6 & 21 & 24 & 6 & 34 & 88 & 17 & 150 & 50 & 32 \\
\hline Microdesmus sp. & 1 & & & & & & & & & & & \\
\hline Acanthurus chirurgus (Bloch, 1787) & & & & 4 & & 12 & 1 & & 2 & & & \\
\hline Achirus achirus (Linnaeus, 1758) & & & & & & & & 3 & & 1 & & 1 \\
\hline Sphoeroides testudineus (Linnaeus, 1758) & & 1 & & & & & & & & 1 & 12 & \\
\hline
\end{tabular}

Tab. III. Lista das espécies de peixes da região entre-marés da Praia do Cabo Branco, João Pessoa, Paraíba, com o número de exemplares coletados entre fevereiro de 1987 e janeiro de 1988 (ponto "B", coletas diurnas).

\begin{tabular}{|c|c|c|c|c|c|c|c|c|c|c|c|c|}
\hline Espécies & Fev & Mar & $\mathrm{Abr}$ & Mai & Jun & Jul & Ago & Set & Out & Nov & Dez & Jan \\
\hline Myrichthys ocellatus (LeSueur, 1825) & & & & & 1 & & & & & & & \\
\hline Ahlia egmontis (Jordan, 1884) & & & & & 2 & & & & & & & \\
\hline Callechelys sp. & & & & & 1 & & & & & & & \\
\hline Lutjanus jocu (Bloch \& Schneider, 1801) & 1 & 1 & 4 & & & & & & & & & \\
\hline Lutjanus apodus (Walbaum, 1792) & & 2 & & & & & & & & & 12 & \\
\hline Abudefduf saxatilis (Linnaeus, 1758) & & 2 & 13 & 1 & 3 & 3 & 2 & 1 & & & & \\
\hline Mugil liza Valenciennes, 1836 & 1 & 2 & 3 & & 1 & 11 & 1 & & & & & \\
\hline Labrisomus nuchipinnis (Quoy \& Gaimard, 1824) & 7 & & 7 & & 5 & & 3 & & & & & \\
\hline Scartella cristata (Linnaeus, 1758) & & & & & & 4 & & & & & & \\
\hline Gobionellus boleosoma (Jordan \& Gilbert, 1882) & & & & & 1 & & & 1 & & & & \\
\hline Bathygobius soporator (Valenciennes, 1837) & 13 & 3 & 15 & 16 & 52 & 88 & 54 & 4 & 3 & 4 & 2 & 11 \\
\hline Acanthurus chirurgus (Bloch, 1787) & & & 1 & & 1 & 5 & & & & & & \\
\hline
\end{tabular}


thygobius soporator), da ordem de $90 \%$. Por outro lado, a recolonização parece ter sido favorecida pela existência de poças adjacentes ou próximas àquelas amostradas, contendo indivíduos não afetados pelo ictiotóxico, sobretudo das espécies gregárias.

Tab. IV. Lista das espécies de peixes da região entre-marés da Praia do Cabo Branco, João Pessoa, Paraiba, com o número de exemplares coletados entre fevereiro de 1987 e janeiro de 1988 (ponto "B", coletas noturnas).

\begin{tabular}{|c|c|c|c|c|c|c|c|c|c|c|c|c|}
\hline Espécies & Fev & Mar & $\mathrm{Abr}$ & Mai & Jun & Jul & Ago & Set & Out & Nov & Dez & Jan \\
\hline Myrophys platyrhinchus Lütken, 1851 & & & & & & & 1 & & & & & \\
\hline Xenomelaniris brasiliensis (Quoy \& Gaimard, 1824) & & & & & & & & & & & & 1 \\
\hline Epinephelus adscencionis (Osbeck, 1771) & & & & & & & & & 1 & & & \\
\hline Lutjanus jocu (Bloch \& Schneider, 1801) & & 6 & & & & & & & & & & \\
\hline Lutjanus apodus (Walbaum, 1792) & & & & 1 & & & & & & 1 & 1 & \\
\hline Stegastes variabilis (Castelnau, 1855) & & 1 & & & & & & & & & & \\
\hline Adudefduf saxatilis (Linnaeus, 1758) & 1 & 2 & 44 & 8 & 6 & 2 & 9 & 1 & 3 & 1 & 1 & 1 \\
\hline Mugil liza Valenciennes, 1836 & & & & & & & 1 & 19 & & & & \\
\hline Mugil gaimardianus Desmarest, 1831 & & & & & & 35 & 1 & & 4 & & & \\
\hline Labrisomus nuchipinnis (Quoy \& Gaimard, 1824) & & & 1 & 4 & 1 & 47 & & & & & & \\
\hline Scartella cristata (Linnaeus, 1758) & & & & 1 & & & 1 & 1 & 2 & & 2 & \\
\hline Bathygobius soporator (Valenciennes, 1837) & 33 & 9 & 1 & & 38 & 2 & 25 & 16 & 30 & 26 & 26 & \\
\hline Sphoeroides testudineus (Linnaeus, 1758) & & & & & & & & & & & & 1 \\
\hline
\end{tabular}

Em estudo realizado na Austrália, LARDNER et al. (1993) encontraram evidências de uma rápida recolonização em poças de maré submetidas ao envenenamento com rotenona (da ordem de 3 a 12 dias), por parte das espécies intertidais, e uma recolonização mais lenta por parte das espécies subtidais. Na recolonização em curto prazo, as espécies gregárias mostraram uma recuperação mais rápida do que as solitárias e crípticas.

No presente estudo, as famílias mais representativas em número de indivíduos capturados (Fig. 4) foram Gobiidae, Pomacentridae e Labrisomidae. As mais representativas em número de espécies (Fig. 5) foram Ophichthidae, Mugilidae e Muraenidae. Estes resultados assemelham-se em parte aos encontrados na Austrália por LARDNER et al (1993), que observaram dominância em número de indivíduos por parte de espécies gregárias, e dominância em número de espécies em grupos de hábitos solitários.

Estudos realizados em outras regiões encontraram, como dominantes em número de indíviduos, as famílias Pomacentridae (LEE 1980), Clinidae (BENNETT 1986), Clinidae e Gobiesocidae (BENNETT \& GRIFFITTS 1984), Cottidae e Gobiesocidae (MORING 1986), e Cottidae (YoshIYAMA 1981; YoshIYAMA et al. 1986).

As famílias dominantes encontradas no presente estudo e em trabalhos realizados em outras áreas, apresentam características morfológicas típicas de peixes da região entre-marés. Isto evidencia a importância das poças para a manutenção de populações de espécies típicas daquela região e de várias espécies costeiras, que utilizam as poças como parte do "home range". Segundo HoRN \& GIBSON (1988), a característica anatômica mais aparente de peixes da região entre-marés é o pequeno tamanho dos indivíduos, que os capacita a ocupar locais onde o risco de serem carregados pelas ondas é reduzido. Este é o caso das espécies de Labrisomidae e Gobiidae, que, além disso, são crípticas. Ainda de acordo com 
HORN \& GIBSON (1988), a habilidade de se manter no lugar é maior em peixes de corpo fino que possuem um perfil achatado ou horizontal. Em duas das famílias encontradas (Gobiidae e Gobiesocidae), esta capacidade é ampliada pela presença de uma ventosa ventral, formada pelas nadadeiras pélvicas modificadas.
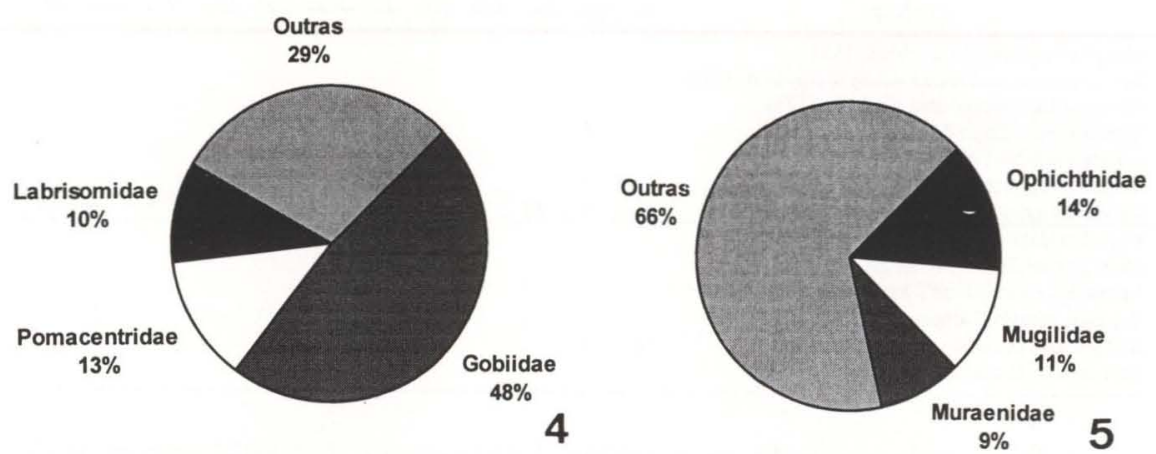

Figs 4-5. Porcentagem das famílias mais representativas em número de indivíduos (4) e em número de espécies (5), capturados na Praia do Cabo Branco, João Pessoa, Paraíba, Brasil.

Com relação à ocorrência nas poças, Bathygobius soporator foi a espécie dominante nos pontos $\mathrm{A}$ e B, tanto nas coletas diurnas quanto noturnas. Esta espécie foi capturada em praticamente todos os meses de coleta, podendo ser considerada como residente primária das poças estudadas. Outras espécies crípticas como Labrisomus nuchipinnis e Gobionellus boleosoma podem ser consideradas como residentes primárias, porém apenas no ponto A. STARKS (1913), em coletas em poças de maré de Natal, Rio Grande do Norte, encontrou Labrisomus nuchipinnis, Salariichthys textilis (=Entomacrodus nigricans ?) e Pomacentrus (=Stegastes) fuscus como espécies mais abundantes.

Abudefduf saxatilis pode ser considerada como residente secundária, nos dois pontos estudados, assim como Lutjanus jocu e L. apodus, porém apenas no ponto A. Foram também capturados jovens de Gymnothorax funebris, Caranx latus, Sparisoma rubripinne, Acanthurus chirurgus e Sphoeroides testudineus, porém como visitantes ocasionais e apenas no ponto A. Jovens e adultos de Myrophis punctatus, Myrichthys ocellatus, Bascanichthys paulensis, Eucinostomus argenteus, E. melanopterus, Stegastes variabilis e Scartella cristata, foram consideradas visitantes ocasionais no ponto A. São visitantes ocasionais do ponto B: jovens de Lutjanus jocu, L. apodus, Mugil liza, M. gaimardianus e jovens e adultos de Labrisomus nuchipinis e Scartella cristata.

Foram consideradas raras: Albula vulpes, Ahlia egmontis, Callechelys sp, Gobiesox strumosus, Mugil trichodon, Microdesmus sp., Sparisoma radians no ponto A, e Myrichthys ocellatus, Ahlia egmontis, Xenomelaniris brasiliensis, Calle- 
chelys sp, Gobionellus boleosoma, Stegastes variabilis e Sphoeroides testudineus no ponto $\mathrm{B}$.

$\mathrm{O}$ índice de diversidade (H') observado no ponto A variou entre 0,45 e 2,25 e entre 0,0 e 1,55 no ponto $B$. $O$ índice médio ( $\left.\mathrm{H}^{\prime}\right)$ ao longo do período de estudo foi de 1,42 para o ponto A e 0,71 para o ponto $\mathrm{B}$. Calculando-se a média mensal (coletas diurnas e noturnas) do índice de diversidade para cada ponto, tentando-se minimizar o efeito da depleção pela rotenona nas amostragens temporalmente próximas, obteve-se uma variação de H' entre 1,34 e 2,29 para o ponto A e entre 0,28 e 1,48 para o ponto B (Fig. 6). Todavia, estes valores são afetados por incluir os indivíduos que eventualmente recolonizaram a poça em curto prazo.

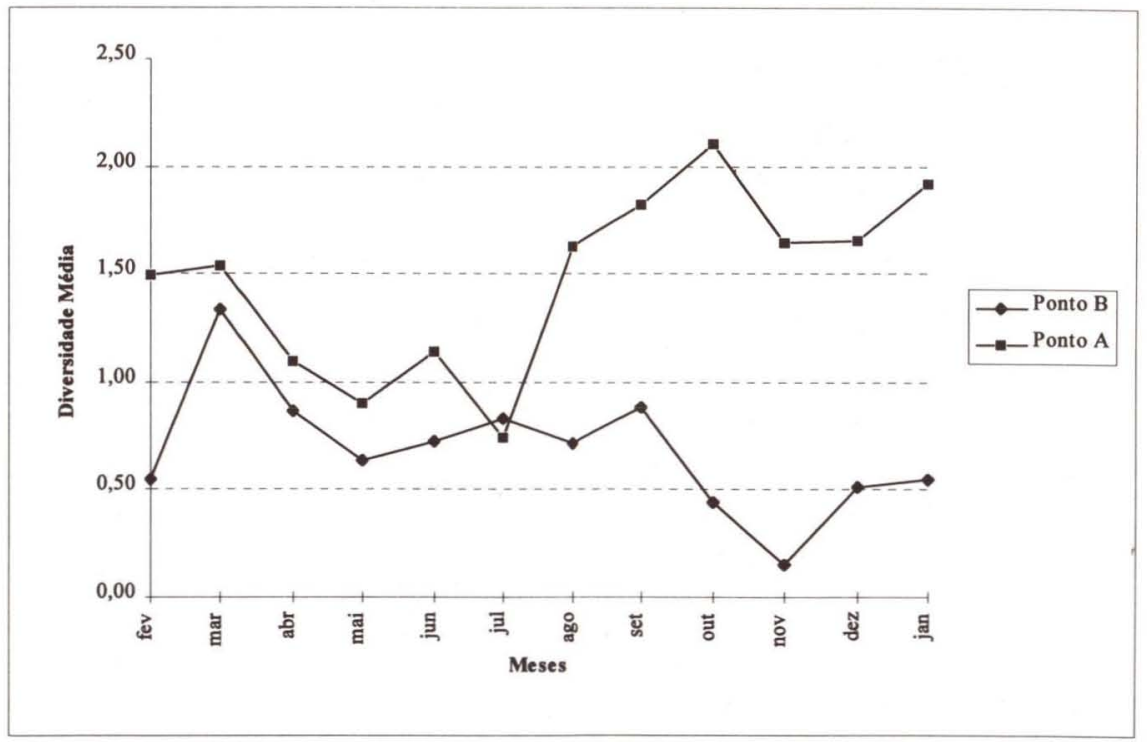

Fig. 6. Diversidade média mensal nos pontos de coleta "A" e "B", Praia do Cabo Branco, João Pessoa, Paraíba, ao longo de todo periodo estudado.

A riqueza de espécies foi maior no ponto $\mathrm{A}$, variando entre oito (fevereiro $\mathrm{e}$ junho) e 23 (julho), para os dados diurnos e noturnos agrupados. No ponto B a riqueza de espécies variou entre três (outubro) e nove (maio), para os dados diurnos e noturnos agrupados A riqueza total de espécies obtida foi de 42 espécies no ponto A e de 18 espécies no ponto $\mathrm{B}$.

A maior diversidade e riqueza de espécies encontradas no ponto $\mathrm{A}$ devem estar associadas à maior área desta poça, que proporciona um maior número de microhabitats quando comparada ao ponto B. O substrato arenoso, por exemplo, local habitado por diversas espécies de Ophichthidae no ponto A, está totalmente ausente no ponto B. A maior área e volume de água do ponto A também proporci- 
onam uma menor yariação de parâmetros ambientais como temperatura e salinidade, que podem atingir níveis críticos em poças de maré. Efetivamente, a temperatura mais elevada da água foi observada no ponto $\mathrm{B}\left(37,5^{\circ} \mathrm{C}\right.$, em novembro). Outro fator que pode comprometer a diversidade e a riqueza no ponto $\mathrm{B}$ é o relativo isolamento destas poças, que apesar de mais próximas da linha de baixamar, encontram-se separadas desta e de outras poças por uma grande extensão de rochas, o que certamente dificulta $o$ acesso dos peixes.

Das 44 espécies obtidas no presente estudo, 34 estavam previamente assinaladas na costa da Paraiba (RosA 1980; RAMOs 1994); as demais representam novos registros de ocorrência: Ahlia egmontis, Myrophis punctatus, Bascanichthys paulensis, Gobiesox strumosus, Lutjanus apodus, Mugil liza, Mugil gaimardianus, Anisotremus surinamensis, Malacoctenus delalandii, e Scartella cristata. Os táxons Mugil sp. e Microdesmus sp. foram representados apenas por alevinos e não puderam ser identificados em nivel específico.

$\mathrm{O}$ grande número de indivíduos jovens obtidos neste trabalho indica a importância das poças de maré estudadas como berçário para diversas espécies de peixes. A crescente utilização das poças por banhistas e pescadores de subsistência poderá comprometer as populações de peixes ali existentes. Estudos periódicos sobre a diversidade da ictiofauna, através de coletas ou censos visuais, devem ser feitos visando o monitoramento desses ambientes. Programas de educação ambiental deverão salientar a importância das poças de maré como áreas de lazer, já que as mesmas constituem-se em verdadeiros aquários naturais.

Material examinado. Abudefduf saxatilis, UFPB 1638, 1654, 2102, 2108, $2116,2122,2237,2245,2249,2262,2269,2277,2285,2293,2296,2306,2325$, 2329, 2339, 2354 2361, 2365, 2375, 2384, 2385, 2412, 2417, 2443, 2452, 2464, 2468, 2579, 2584, 2587; Acanthurus chirurgus, UFPB 2115, 2253, 2291, 2301, 2323, 2374, 2583; Achirus achirus, UFPB 2341, 2419, 2455; Ahlia egmontis, UFPB 1649, 2266, 2286, 2337; Albula vulpes, UFPB 2376, 2390, 2400, 2466; Anisotremus surinamensis, UFPB 1647, 2107; Bascanichthys paulensis, UFPB 2219, 2314, 2347, 2370, 2414, 2478; Bathygobius soporator, UFPB 1636, 1637, 1659, 2103, 2104, 2117, 2121, 2223, 2226, 2233, 2236, 2244, 2248, 2251, 2265, 2268, 2271, $2278,2288,2294,2298,2307,2326,2328,2333,2338,2352,2364,2367,2371$, 2383, 2388, 2395, 2398, 2418, 2424, 2432, 2440, 2444, 2451, 2456, 2470, 2479, 2580, 2581, 2586, 2589; Scartella cristata, UFPB 1642, 1661, 2132, 2260, 2263, 2264, 2276, 2283, 2299, 2330, 2353, 2381, 2403, 2426, 2441, 2582; Callechelys sp, UFPB 2280; Caranx latus, UFPB 2401; Coryphopterus glaucofrenum, UFPB 2428; Epinephelus adscensionis, UFPB 2133, 2350, 2380, 2399; Eucinostomus argenteus, UFPB 2324, 2332, 2397, 2434, 2461; Eucinostomus melanopterus, UFPB 2258, 2348, 2396, 2439, 2458, 2474; Gobiesox strumosus, UFPB 1641, 1663 , 2356; Gobionellus boleosoma, UFPB 1648, 1662, 2109, 2114, 2131, 2228, 2242, 2290, 2358, 2366, 2377, 2391, 2410, 2422, 2448, 2460, 2467; Gymnothorax funebris, UFPB 1639, 2230, 2243, 2257, 2259, 2409, 2420, 2593; Gymnothorax moringa, UFPB 2313; Gymnothorax vicinus, UFPB 1665, 2111, 2317, 2411, 2427; Haemulon steindachneri, UFPB 2309; Holocentrus ascensionis, UFPB 2415; Labrisomus nuchipinnis, UFPB 1645, 1653, 2110, 2118, 2120, 2222, 2231, 2243, 
2261, 2272, 2279, 2284, 2302, 2305, 2310, 2349, 2393, 2438, 2449, 2471, 2480, 2590, 2591; Lutjanus apodus, UFPB 2227, 2240, 2246, 2252, 2256, 2259, 2274, $2304,2315,2332,2345,2379,2394,2413,2416,2437,2442,2446,2457,2475$; Lutjanus jocu, UFPB 1644, 1664, 2102, 2112, 2119, 2225, 2229, 2235, 2239, 2255, 2270, 2300, 2327, 2389, 2408, 2323, 2425, 2447, 2477, 2578; Malacoctenus delalandii, UFPB 1643, 1660, 2289, 2577; Mugil curema, UFPB 2316, 2346, 2373; Mugil gaimardianus, UFPB 2320, 2331, 2336, 2360, 2369, 2382, 2387, 2588; Mugil liza, UFPB 1669, 2113, 2234, 2247, 2250, 2292, 2295, 2308, 2355, 2392, 2469, 2585; Mugil trichodon, UFPB 2402, 2435; Mugil sp., UFPB 2303, 2378; Myrichthys ocellatus, UFPB 1646, 2254, 2282, 2287, 2321, 2335, 2357, 2407, 2436, 2445, 2454, 2499; Myrophis punctatus, UFPB 2106, 2281, 2297, 2322, 2351, 2372, 2386, 2405, 2429, 2453, 2472; Polydactylus virginicus, UFPB 2312, 2340, 2368; Sparisoma radians, UFPB 1666; Sparisoma rubripinne, UFPB 1640, 2105, 2241, 2275, 2311, 2342, 2363, 2430, 2476; Sphoeroides testudineus, UFPB 1658, 2362, 2404, 2431, 2450, 2463, 2473; Stegastes variabilis, UFPB 2238, 2273, 2319, 2421, 2465; Trachinotus falcatus, UFPB 2406.

AGRADECIMENTOS. A Alba M. Almeida e Angelo J. Sales pela participação nas atividades de campo e laboratório; a Cláudia R.R. Nunes pelo apoio no laboratório e na datilografia do texto, e a Amélia I. Kanagawa pela identificação do material botânico. Somos ainda gratos a Luiz Gonzaga S. Jr., Marcelo M. Oliveira, Robson T. C. Ramos e Ruth Silva, pela ajuda eventual nas coletas.

\section{REFERÊNCIAS BIBLIOGRÁFICAS}

ALmEIDA, V.G. 1973. New records of tidepool fishes from Brazil. Pap. Avuls Zool., São Paulo, 26 (14): 187-191.

BAKUS, G.J. 1990. Quantitative Ecology and Marine Biology. Rotterdam., A.A. Balkema, $157 \mathrm{p}$.

BECKLEY, L.E. 1985. The fish community of East Cape tidal pools and an assessment of the nursery function of this habitat. S. Afr. J. Zool. 20 (1): 21-27.

BENNETT, B.A. 1986. The rock-pool fish community of Koppie Alleen, South Africa and an assesment of the importance of Cape rock pool as nurseries for juvenile fish. S. Afr. J. Zool. 22 (1): 25-32.

BenNetT, B.A. \& C.L. Griffiths. 1984. Factors affecting the distribution, abundance and diversity of rock-pool fishes on the Cape Peninsula, South Africa. S. Afr. J. Zool. 19 (2): 97-104.

CRABTREE, R.E. \& J.M. DEAN. 1982. The structure of two South Carolina estuarine tidal pool fish assemblages. Estuaries 5 (1): 2-9.

DoTY, M.S. 1957. Rocky intertidal surfaces, p. 335-385. In: J.W. HEDGPETH (Ed.). Treatise on marine ecology and paleoecology. Ecology. Boulder, The Geological Society of America, Vol. 1, VIII+1296p.

Fitch, J.E. \& R.J. LAVENBERG. 1975. Tidepool and nearshore fishes of California. Berkeley, University of California Press, 156p. 
HoRN, M.H. \& R.N. GiBSON. 1988. Intertidal fishes. Sci. Amer. 258 (1): 54-60. KRICHER, J.C. 1972. Bird Species Diversity: The effect of species richness and equitability on the diversity index. Ecology 53 (2): 278-282.

LARDNER, R.; W. IVANTSOFF \& L.E.L.M. Crowley. 1993. Recolonization by fishes of a rocky intertidal pool following repeated defaunation. Australian Zool. 29 (1-2): 85-92.

LEE, S.C. 1980. Intertidal fishes of a rocky pool of the Sanhsientai, Eastern Taiwan.

Bull. Inst. Zool. Acad. Sin. 16 (1): 19-26.

LUDWIG, J.A. \& J.F. REYNOLDS. 1988. Statistical Ecology: a primer on methods and computing. New York, John Wiley \& Sons, 337p.

MARGALEF, R. 1986. Ecologia. Barcelona, Ediciones Omega S.A., 951p.

MORING, J.R. 1986. Seasonal presence of tidepool fish species in a rocky intertidal zone of northern California, USA. Hydrobiologia 134: 21-27.

NELSON, J.S. 1994. Fishes of the world. New York, John Wiley \& Sons, $3^{\text {rd }}$ ed., $\mathrm{XVII}+600 \mathrm{p}$.

PIEPENBURG, D. \& U. PIATKOWSKI. 1992. A program for computer-aided analyses of ecological field data. Cabios 8 (6): 587-590.

RAmos, R.T.C. 1994. Análise da composição e distribuição da fauna de peixes demersais da plataforma continental da Paraíba e estados vizinhos. Rev. Nordestina Biol. 9 (1): 1-30.

RANDALL, J.E. 1963. Methods of collecting small fishes. Underwater Naturalist 1 (2): 6-11, 32-36.

RosA, R.S. 1980. Lista sistemática de peixes marinhos da Paraíba (Brasil). Rev. Nordestina Biol. 3 (2): 205-226.

STARKS, E.C. 1913. The fishes of the Stanford Expedition to Brazil. Stanford, Stanford University, 77p.

THORne-Miller, B.L. \& J.G. CATENA. 1991. The living ocean: Understanding and protecting marine biodiversity. Washington, Island Press, 180p.

YoshIYAMA, R.M. 1981. Distribution and abundance patterns of rocky intertidal fishes in central California. Env. Biol. Fish. 6 (3/4): 315-332.

Yoshiyama, R.M.; C. SASSAMan \& R.N. Lea. 1986. Rocky intertidal fish communities of California, USA: Temporal and spatial variation. Environ. Biol. Fishes 17 (1): 23-40.

Recebido em 25.VII.1996; aceito em 06.V.1997. 